\title{
Is fibromyalgia accompanying Behçet's disease more severe than primary fibromyalgia?
}

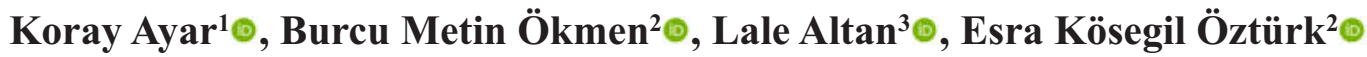 \\ ${ }^{1}$ Department of Internal Medicine, Division of Rheumatology, University of Health Sciences, Bursa Yüksek Ihtisas Training and Research \\ Hospital, Bursa, Turkey \\ ${ }^{2}$ Department of Physical Medicine and Rehabilitation, University of Health Sciences, Bursa Yüksek Ihtisas Training and Research \\ Hospital, Bursa, Turkey \\ ${ }^{3}$ Department of Physical Medicine and Rehabilitation, Uludağ University School of Medicine, Bursa, Turkey
}

\section{ABSTRACT}

Objectives: Although there are studies in the literature about how Behçet's disease is affected in the presence of fibromyalgia, there is no information about how the severity of fibromyalgia is in the presence of Behçet's disease. This study aims to evaluate the severity of fibromyalgia by comparing the impact of fibromyalgia and pain thresholds between fibromyalgia patients with and without Behçet's disease.

Methods: Twenty-five fibromyalgia patients with Behçet's disease and 34 primary fibromyalgia patients were included in this cross-sectional study. All participants completed the fibromyalgia impact questionnaire, and pain thresholds were measured at 18 tender points using algometry.

Results: The mean fibromyalgia impact questionnaire scores in fibromyalgia patients with and without Behçet's disease were $66.6 \pm 18.4$ and $60.4 \pm 14.5$, respectively $(p=0.157)$. Pain thresholds were not different between the groups in 15 of the 18 tender points (TP). In fibromyalgia patients with Behçet's disease, pain thresholds in TP-15, TP-16 and TP-17 were $11.44 \pm 4.84,11.92 \pm 5.00$ and $15.16 \pm 4.89$ pounds, respectively, while in primary fibromyalgia patients those were $8.41 \pm 2.68,8.14 \pm 2.76$ and $12.14 \pm 4.06$ pounds respectively. Pain thresholds in TP-15, TP-16 and TP-17 were significantly different between the groups $(p=0.003, p=0.003$, $p=0.014$, respectively).

Conclusions: According to the literature data, although fibromyalgia has an effect on Behçet's disease, especially in cases associated with central sensitization syndromes, the severity of fibromyalgia was not found to be different in fibromyalgia patients with and without Behçet's disease in this study. There may be a relationship between Behçet's disease and fibromyalgia due to non-inflammatory causes such as central sensitization.

Keywords: Behçet's disease, fibromyalgia, pain thresholds

$\mathrm{F}$ ibromyalgia (FM) is a common cause of chronic widespread musculoskeletal pain, often accompanied by fatigue, cognitive disturbance, psychiatric symptoms, and multiple somatic symptoms. There are many factors affecting the severity of FM. These include; working status, coping ability, mood disor- ders, eveningness, inflammation, arterial stiffness, education, and exercise status [1-8]. The majority of the factors affecting the severity of FM are factors that are not based on inflammation. However, in recent studies, it has been found that inflammation-related conditions such as obesity, arterial stiffness, IL-6, and 
IL-8 levels affect the severity of FM [4, 6, 9]. There are also studies showing that FM is associated with inflammation markers, systemic inflammation, and neuroinflammation $[10,11]$. Moreover, the frequency of FM in many diseases associated with inflammation is higher than the frequency of FM in the healthy population [12-18]. Although the relationship between $\mathrm{FM}$ and inflammation is increasing, and FM is more common in chronic inflammatory diseases, there is not much known about how inflammatory diseases have effects on FM.

Behçet's disease (BD) is an inflammatory disease characterized by recurrent oral aphthous ulcers and numerous potential systemic manifestations. The incidence of FM in BD is higher than in the healthy population, and its frequency ranges from $5.7 \%$ to $37.1 \%$ [19]. The presence of FM has a negative effect on depression, anxiety, sleep quality, and quality of life in patients with BD [19-21]. However, there is no literature information about how FM is effected in the presence of BD. The aim of this study is to evaluate the severity of FM by comparing pain thresholds and FIQ scores between FM patients with and without BD.

\section{METHODS}

\section{Study design}

This cross-sectional study was conducted between March 2018 and February 2019. The study protocol was approved by the local Ethics Committee (2011KAEK-25 2018/03-14), and written informed consents were obtained from each participant. The study was conducted in accordance with the principles of the Declaration of Helsinki.

\section{Selection of the participants}

Behçet patients included in this study were selected among patients who met the international classification criteria [22] and were being followed up from the rheumatology outpatient clinic of an education and research hospital, and primary FM patients were selected among the patients followed from the physical therapy and rehabilitation outpatient clinic of the same hospital. Volunteers aged 18-75 were included in this study. A detailed physical examination of all volunteers was performed, and previously performed laboratory tests were examined from electronic medical records, and the medications they used were questioned.

\section{Inclusion and exclusion criteria}

Those with a previously known systemic rheumatic or comorbid disease, pregnancy, and depression were excluded from this study. In addition, other than those used in the treatment of BD, those with chronic drug use were not included in the study.

\section{Screening of fibromyalgia}

After the first evaluation, the presence of FM in all participants were screened according to the 2013 American College of Rheumatology (ACR) alternative criteria [23]. Alternative criteria were derived from the Symptom Impact Questionnaire (SIQR) symptoms and the 28-area pain location inventory (PLI). In SIQR symptoms, each of the intensities of the typical ten symptoms of fibromyalgia over the last seven days was graded over 10 points by the participants. The symptom impact questionnaire symptoms score was obtained as half of the total score obtained from the SIQR. In PLI, the participants were asked about the 28 specific localizations of the body in which they defined persistent pain in the last seven days. The total number of painful localizations is the pain location score of the patient. A participant fulfilling the following was defined as having FM: SIQR symptom score $\geq 21$ and pain location score $\geq 17$. Those with FM according to alternative criteria were included in the study.

Assessment of the participants included in the study

In order to determine the severity of FM in the participants, all participants included in the study filled the FM impact questionnaire (FIQ), and the pain thresholds of all participants were measured.

\section{Fibromyalgia Impact Questionnaire (FIQ)}

FIQ is a self-filled test that quantitatively evaluates the functional status, stiffness and pain level, anxiety, and depression status of patients with FM, which was first described in 1991 by Burckhardt et al. [24]. FIQ is the most commonly used test to detect the severity of FM. All participants completed the validated Turkish version of the FIQ under the supervision of a researcher [25]. FIQ is composed of 10 items; the 
first item consists of sub-items that question how easily some activities in normal daily life can be performed and the responses given to each sub-item were evaluated between 0 (always able to do) and 3 points (never able to do) according to the Likert scale. The second item questions how many days the participant feels good in the last one week, and scoring is based on the number of days he feels bad. The third item questions how many days of the week, the participant is unable to work due to FM. The scores obtained from each of the first three items were standardized according to the 0-10 scale and were then included in the scoring. In the other seven items that were evaluated for scoring, participants evaluated the severity of work status, pain, fatigue, well being, morning stiffness, anxiety, and depression between 0 10 on the Likert scale. FIQ score is the sum of the scores obtained from the ten items (0-100).

\section{Assessment of pain thresholds}

In this study, the pain thresholds were determined using dolorimeter at the tender points (TPs) defined in the ACR 1990 classification criteria defined for FM [26]. These TPs consist of 18 points, in which nine sensitive point regions are evaluated on both sides (Fig. 1). These nine sensitive point regions are as follows; suboccipital muscle insertions (TP-1, TP2), anterior aspects of the intertransverse spaces at C5-C7 (TP-11, TP-12), the midpoint of the upper border of the trapezius muscle (TP-5, TP-6), origins of supraspinatus muscle (TP-3, TP-4), second costochondral junctions (TP-13, TP-14), lateral epicondyles (TP-15, TP-16), upper outer quadrants of buttocks in anterior fold of muscle (TP-7, TP-8), posterior to the trochanteric prominence (TP-9, TP-10), medial fat pad proximal to the knee joint line (TP-17, TP-18). Dolorimeter is an instrument that objectively measures pain threshold and pain tolerance. The dolorimeter used in this study (Baseline ${ }^{\circledR}$ Dolorimeters, New York/USA) consists of a metal piston with a round rubber disc about $1 \mathrm{~cm}$ in diameter attached to a dial measuring pressures in pounds (Lbs). The user can apply the desired case pressure by holding the handset. Investigators advance the instrument at a rate of approximately 6-8 Lbs per second and instruct the patient to "tell me when this becomes painful." The initial pressure at which the patient felt pain was recorded in pounds. The procedure was repeated three times at intervals of 60 seconds to evaluate the mean pound pressure pain threshold.

\section{Comparisons between groups}

Demographic characteristics, FIQ total scores, and pain thresholds measured at the tender points with the dolorimeter were compared between the groups.

\section{Statistical Analysis}

SPSS Version 16.0 for Windows (SPSS Inc,
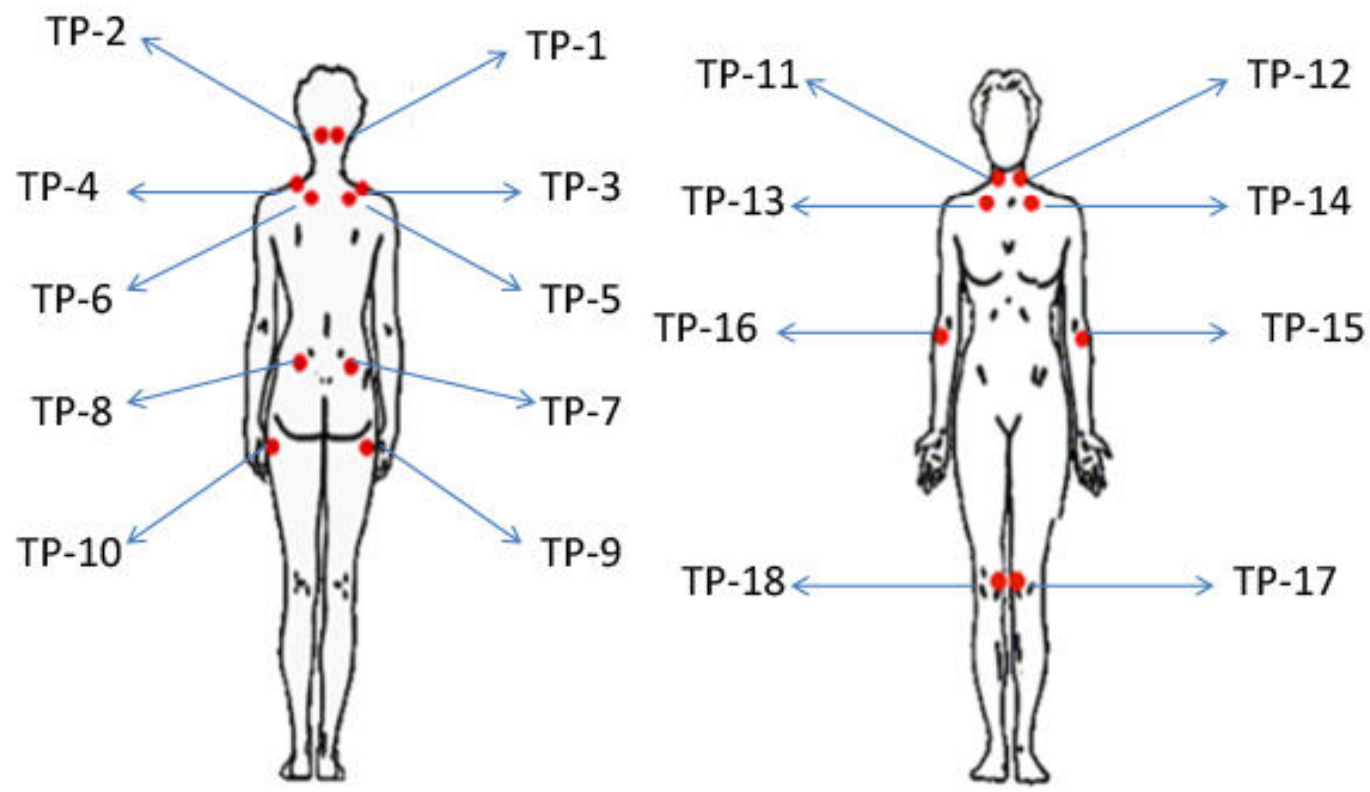

Fig. 1. The tender points where pain threshold levels were measured with a dolorimeter. 
Chicago, IL, USA) was used to analyze all data statistically. The data had previously been subjected to a normal distribution test (Kolmogorov-Smirnov). In comparing the quantitative variables, student's t-test was used for the normally distributed variables, and Mann-Whitney U-test was used for the variables that were not normally distributed. For the comparison of qualitative data, Fisher's $\chi^{2}$ or $\chi^{2}$ test was used. All tests were two-tailed, and $p$ values $<0.05$ were considered to indicate statistical significance.

\section{RESULTS}

Ninety-nine participants from the study group, 34 participants from the control group were screened for alternative criteria with fibromyalgia, and 25 participants (19 females, six male) from the study group, and 34 participants ( 28 females, six male) from the control

Table 1. Disease-specific features and drugs of patients with Behcet's disease with fibromyalgia

\begin{tabular}{|lc|}
\hline & $\begin{array}{c}\text { Patients with } \\
\text { BD with FM } \\
(\mathbf{n}=\mathbf{2 5})\end{array}$ \\
\hline Disease specific features, $\mathbf{n}(\%)$ & \\
\hline Oral ulcerations & $25(100)$ \\
\hline Genital ulcerations & $19(76)$ \\
\hline Erythema nodosum & $17(68)$ \\
\hline Acneiform lesions & $19(76)$ \\
\hline Pathergy & $12(48)$ \\
\hline Eye lesions & $4(16)$ \\
\hline Venous disease & $3(12)$ \\
\hline Arterial disease & $1(4)$ \\
\hline $\begin{array}{l}\text { Neurologic disease } \\
\text { (Parenchymal) }\end{array}$ & $3(12)$ \\
\hline Drugs, $\mathbf{n}$ (\%) & $15(60)$ \\
\hline Colchicine & $10(40)$ \\
\hline Corticosteroid & $3(12)$ \\
\hline Azathioprine & $1(4)$ \\
\hline Infliximab & $8(32)$ \\
\hline $\begin{array}{l}\text { No medication (newly } \\
\text { diagnosed) }\end{array}$ & \\
\hline
\end{tabular}

$\mathrm{BD}=$ Behçet's disease, $\mathrm{FM}=$ fibromyalgia group were included in the study. Disease-specific features and drugs of patients with BD with FM are shown in Table1. Human leukocyte antigen (HLA)B51 positive in $14(56.0 \%)$ of Behçet's patients. The comparison of demographic data, PLI, SIQR symptoms, and FIQ total scores of the study and control groups are shown in Table 2. The mean age of patients with BD with FM was $40.0 \pm 10.6$ years, and the mean age of patients with primary FM was $44.3 \pm 8.9$ years. Age and gender were not different between the groups ( $p=0.095, p=0.549$, respectively). Mean PLI and median SIQ scores were not different between groups ( $p=0.636, p=0.589$, respectively). The mean FIQ scores were $66.6 \pm 18.4$ in the study group, $60.4 \pm$ 14.5 in the control group, and the difference between the groups was not statistically significant ( $p=0.157$ ). A comparison of pain thresholds in patients with BD with FM and primary FM patients are shown in Table 3. When compared for each TP individually, the pain thresholds between the groups were not different, except for three of the TPs. The median pain thresholds in TP-15 and TP-16 were 10.0 (minimum-maximum: 3-20 Lbs) and 12.0 Lbs (minimum-maximum: 4-20 Lbs), respectively in patients with BD with FM, and 8.0 (minimum-maximum: 4$14 \mathrm{Lbs}$ ) and $8.0 \mathrm{Lbs}$ (minimum-maximum: 4-14 Lbs), respectively in primary FM patients. Pain thresholds in TP-15 and TP-16 were significantly different between the groups ( $p=0.003, p=0.003)$. The median pain thresholds in TP-17 were 14.0 (minimum-maximum: 7-24 Lbs) in patients with $\mathrm{BD}$ with FM, and 11.0 (minimum-maximum: 7-22 Lbs) in primary FM patients. Pain thresholds in TP-17 were significantly different between the groups $(p=0.014)$.

\section{DISCUSSION}

In this study, we compared the severity of FM in terms of FIQ scores and pain thresholds in FM patients with and without $\mathrm{BD}$, and we did not find the severity of FM generally different between the groups. To the best of our knowledge, there is no study in the literature investigating the effect of $\mathrm{BD}$ on the severity of FM, and this is the first study to demonstrate that disease severity is not different in FM patients with and without BD. Very limited data are available on the effects of rheumatic diseases on FM. In the study of 
Tablo 2. Comparison of demographic data, fibromyalgia impact questionnaire scores and total number of tender points between patients with primary fibromyalgia and patients with Behçet's disease with fibromyalgia

\begin{tabular}{cccc}
\hline & $\begin{array}{c}\text { Patients with BD with FM } \\
(\mathbf{n}=\mathbf{2 5})\end{array}$ & $\begin{array}{c}\text { Primer FM } \\
(\mathbf{n = 3 4 )}\end{array}$ & $p$ value \\
\hline Female, n (\%) & $19(76.0)$ & $28(82.4)$ & 0.549 \\
Age, years & $40.0 \pm 10.6$ & $44.3 \pm 8.9$ & 0.095 \\
PLI & $32.7 \pm 5.9$ & $33.6 \pm 7.3$ & 0.636 \\
SIQ & $21.0(16-28)$ & $19.5(17-28)$ & 0.589 \\
FIQ & $66.6 \pm 18.4$ & $60.4 \pm 14.5$ & 0.157 \\
\hline
\end{tabular}

$\mathrm{BD}=$ Behçet's disease, FMS = fibromyalgia syndrome, PLI = pain location inventory, SIQ = symptom impact questionnaire, FIQ = fibromyalgia impact questionnaire, $\mathrm{TPs}=$ tender points

Tablo 3. Comparison of pain thresholds in patients with Behçet's disease with fibromyalgia and primary fibromyalgia patients

\begin{tabular}{|c|c|c|c|c|c|c|c|}
\hline \multirow[b]{2}{*}{ pounds } & \multicolumn{3}{|c|}{$\begin{array}{l}\text { Patients with BD with FM } \\
\qquad(\mathrm{n}=25)\end{array}$} & \multicolumn{3}{|c|}{$\begin{array}{c}\text { Primer FM } \\
(\mathbf{n}=\mathbf{3 4}) \\
\end{array}$} & \multirow[t]{2}{*}{$p$ value } \\
\hline & Mean \pm SD & median & $\min -\max$ & Mean \pm SD & median & $\min -\max$ & \\
\hline TP-1 & $9.64 \pm 4.27$ & 8.0 & $5-20$ & $10.00 \pm 3.01$ & 10.0 & 6-16 & 0.275 \\
\hline TP-2 & $9.28 \pm 3.93$ & 8.0 & $4-18$ & $9.70 \pm 3.12$ & 10.0 & $5-19$ & 0.426 \\
\hline TP-3 & $12.72 \pm 5.2$ & 14.0 & $4-28$ & $11.67 \pm 3.55$ & 11.0 & $6-20$ & 0.392 \\
\hline TP-4 & $12.84 \pm 5.47$ & 13.0 & $5-26$ & $10.91 \pm 3,26$ & 10.0 & $4-18$ & 0.097 \\
\hline TP-5 & $12.80 \pm 4.89$ & 13.0 & $4-22$ & $11.97 \pm 3.28$ & 12.0 & $7-21$ & 0.440 \\
\hline TP-6 & $13.08 \pm 5.52$ & 13.0 & $4-24$ & $11.79 \pm 3.35$ & 12.0 & $5-18$ & 0.272 \\
\hline TP-7 & $14.68 \pm 5.20$ & 14.0 & $6-26$ & $14.38 \pm 4.87$ & 14.0 & $5-29$ & 0.823 \\
\hline TP-8 & $16.00 \pm 5.25$ & 16.0 & $7-26$ & $14.32 \pm 4.39$ & 13.5 & $8-26$ & 0.188 \\
\hline TP-9 & $15.20 \pm 4.98$ & 15.0 & $5-24$ & $14.14 \pm 3.97$ & 13.5 & $7-24$ & 0.371 \\
\hline TP-10 & $14.36 \pm 4.60$ & 15.0 & $4-24$ & $13.05 \pm 4.45$ & 12.5 & $6-26$ & 0.279 \\
\hline TP-11 & $4.96 \pm 1.48$ & 6.0 & $3-8$ & $5.35 \pm 1.63$ & 6.0 & $3-11$ & 0.432 \\
\hline TP-12 & $5.44 \pm 1.98$ & 6.0 & $3-8$ & $5.55 \pm 1.95$ & 5.0 & $3-11$ & 0.882 \\
\hline TP-13 & $7.12 \pm 3.01$ & 7.0 & $2-14$ & $8.02 \pm 2.43$ & 8.0 & $3-13$ & 0.203 \\
\hline TP-14 & $7,44 \pm 3.24$ & 7.0 & $3-14$ & $8.29 \pm 2.38$ & 8.0 & $3-15$ & 0.114 \\
\hline TP-15 & $11.44 \pm 4.84$ & 10.0 & $3-20$ & $8.41 \pm 2.68$ & 8.0 & $4-14$ & $0.003^{*}$ \\
\hline TP-16 & $11,92 \pm 5.00$ & 12.0 & $4-20$ & $8.14 \pm 2.76$ & 8.0 & $4-14$ & $0.003^{*}$ \\
\hline TP-17 & $15.16 \pm 4.89$ & 14.0 & $7-24$ & $12.14 \pm 4.06$ & 11.0 & $7-22$ & $0.014^{*}$ \\
\hline TP-18 & $13.00 \pm 3.92$ & 12.0 & $6-22$ & $12.55 \pm 4.13$ & 12.0 & $6-21$ & 0.579 \\
\hline
\end{tabular}

$\mathrm{BD}=$ Behçet's disease, $\mathrm{FM}=$ fibromyalgia, $\mathrm{TP}=$ tender point, $\min =$ minimum, $\max =$ maximum, $\mathrm{SD}=$ standard deviation, ${ }^{*} p<0.05$

De Araújo et al., the FIQ scores of FM patients with and without systemic lupus erythematosus (SLE) were compared, and no significant difference was found between the groups [27]. In a prospective study conducted by Jiao et al. [28], FM patients with and without rheumatic diseases (undifferentiated inflam- 
matory arthritis, rheumatoid arthritis, undifferentiated connective tissue disease, Sjögren's syndrome, SLE, polymyalgia rheumatic, psoriatic arthritis, reactive arthritis, antiphospholipid antibody syndrome) were evaluated prospectively before the fibromyalgia treatment program (FTP) began, and 6 and 12 months after the FTP. As a result, they found that after the FTP, FM patients with rheumatic diseases improved FIQ subscales less than those without rheumatic diseases [28]. In this study, similar to the study conducted by De Araújo et al. [27], the FIQ scores were not different in FM patients with and without BD. However, since the treatment response was not evaluated in this study, we do not know how the presence of BD affects the treatment response in FM.

FIQ is the most commonly used test to measure the severity of disease in FM patients, but whether it is indicative of inflammation is not clear. In some studies, it was found to be associated with indicators of inflammation in FM, while in some studies, it was not correlated with the markers of inflammation [9, 29, 30]. When Gunturk et al. [29] evaluated the parameters of arterial stiffness in FM patients, they found that the parameters of arterial stiffness were highly correlated with the FIQ score. However, in the study of Ranzolin et al. [30], there was no correlation between biomarkers such as IL-6, IL-8, IL-10, TNFalpha, and FIQ scores in FM patients. Since BD is a systemic inflammatory disease and FM has also found to be associated with inflammation in recent years, we assumed that BD might have an impact on the severity of FM. However, we found that the disease severity of FM patients evaluated by FIQ did not differ according to the presence of BD. Since we do not know to what extent FIQ scores are associated with inflammation in FM in this study, whether there is an association on the basis of inflammation between these two diseases can only be determined by studies that quantitatively investigate inflammatory markers when both diseases are together and not together.

Measurement of pain threshold is another method used in the quantitative evaluation of disease severity in FM. In general, the pain threshold in fibromyalgia is lower than the healthy population, and the main reason for this is exaggerated modulation of the pain stimulus delivered to the brain in FM patients, which is described as central sensitization [31]. Of course, this is true for patients who assume no pathology in the pathways, such as peripheral nerves, where the pain stimulus is delivered to the brain. There is very limited information in the literature about how pain thresholds are affected in FM patients, depending on whether they are accompanied by a rheumatic disease. In a single study from Ostuni et al. [32], algometry values were compared between FM patients with and without Sjögren syndrome, and algometry values were found to be lower in primary FM patients. In this study, although the pain thresholds were found to be low in primary FM patients in a few regions as in the study of Ostuni et al. [32], in contrast, pain thresholds were not different in the majority of the regions where the assessment was performed. Painful stimulus arising in the periphery is received by specialized nociceptors, and peripheral nociceptive stimulus is transmitted to the central nervous system through the dorsal horn of the spinal column. Finally, after modulating the painful stimulus in the central nervous system, the painful stimulus is perceived by the person. Disorders in the modulation of the stimulus transmitted to the central nervous system, as well as the pathologies that may affect the transmission of the impulse in the peripheral nerves can affect the perceived severity of the painful stimulus. For example, in cases such as central sensitization that causes the amplification of the painful stimulus in the central nervous system, the painful stimulus can be exaggerated, and the pain threshold may decrease as in FM [31]. In cases where peripheral nerves are affected, such as polyneuropathy due to any disease, the pain threshold may increase as the transmission rate will be affected [33]. Central nervous system involvement can be seen in both BD and Sjögren's syndrome [34, 35]. We do not know whether there is peripheral nerve involvement in BD with the literature information so far; however, in Sjögren's syndrome, peripheral nerves are frequently affected [36]. In the study of Ostuni et al. [32], higher pain thresholds in the presence of Sjögren's syndrome in FM patients may be related to the effect of Sjogren's syndrome on the peripheral nerves. In this study, the fact that pain thresholds were not different from primary FM patients in FM patients with BD may be due to the fact that peripheral nerves were not affected much in BD. However, in order to test the accuracy of this hypothesis, there is a need for controlled studies in Behçet patients, in which pain thresholds are measured simultaneously with elec- 
tromyography tests.

Although the effect of BD on FM has not been studied, there are some studies evaluating the effect of FM on BD. The effects of FM on disease activity in BD are contradictory, and there are studies showing that disease activity in BD did not change in the presence of FM, as well as studies showing that disease activity was increased $[19,20]$. In these studies, when the activity of BD was evaluated using a measurement method such as BD current activity form, which includes parameters such as fatigue, joint pain, and headache, which can be seen frequently in FM, disease activity in BD was found to be high in the presence of FM [37]. However, when disease activity was evaluated according to more systemic inflammation-related parameters such as erythrocyte sedimentation rate, $\mathrm{C}$-reactive protein, and clinical activity index, the presence of FM was not associated with disease activity in patients with BD [20]. The effect of FM on BD may be related to the effects of central sensitization related conditions such as pain, fatigue, depression, and anxiety on BD rather than affecting systemic inflammation. As a matter of fact, in the study of Lee et al. [20], anxiety and depression scores were higher in Behçet's patients who were accompanied by FM than those who did not. Since the disease most closely associated with central sensitization is FM, the effect of FM on BD can be expected to be higher than the effect of BD on FM. The results of this study support this hypothesis. However, more comprehensive and prospective studies are needed to clarify this issue.

\section{Limitations}

This study was conducted in a limited number of patient populations, and further studies are needed to clarify this issue. The disease activity of fibromyalgia was assessed through self-assessed questionnaires and pain thresholds. Perhaps, evaluating arterial stiffness or studying some inflammation markers like IL-6 or IL-8 could provide more objective information about the effect of BD on FM. The fact that we did not investigate disease activity in FM by using objective markers is one of the shortcomings of the study. One investigator made the measurements of pain thresholds. In this study, conditions that may have the potential to affect the severity of FM, including; coping, depression, socioeconomic level, and exercise status, were not compared between the groups. The most important limitation of this study is that the participants included in the study were not evaluated for other conditions that might affect the severity of FM.

\section{CONCLUSION}

The results of this study show that the presence of BD does not affect the severity of FM. However, according to literature data, the clinical conditions associated with central sensitization in patients with BD were more severe in the presence of FM. There may be a relationship between $\mathrm{BD}$ and FM due to non-inflammatory causes such as central sensitization.

\section{Conflict of interest}

The authors disclosed no conflict of interest during the preparation or publication of this manuscript.

\section{Financing}

The authors disclosed that they did not receive any grant during conduction or writing of this study.

\section{REFERENCES}

1. Gowans SE, deHueck A, Voss S, Richardson M. A randomized, controlled trial of exercise and education for individuals with fibromyalgia. Arthritis Care Res 1999;12:120-8.

2. Goldenberg DL, Mossey CJ, Schmid CH. A model to assess severity and impact of fibromyalgia. J Rheumatol 1995;22:23138.

3. Rooks DS, Silverman CB, Kantrowitz FG. The effects of progressive strength training and aerobic exercise on muscle strength and cardiovascular fitness in women with fibromyalgia: a pilot study. Arthritis Rheum 2002;47:22-8.

4. Lee JH, Cho KI, Kim SM, Lee HG, Kim TI. Arterial stiffness in female patients with fibromyalgia and its relationship to chronic emotional and physical stress. Korean Circ J 2011;41:596-602.

5. Consoli G, Marazziti D, Ciapparelli A, Bazzichi L, Massimetti G, Giacomelli C, et al. The impact of mood, anxiety, and sleep disorders on fibromyalgia. Compr Psychiatry 2012;53:962-7. 6. Mendieta D, De la Cruz-Aguilera DL, Barrera-Villalpando MI, Becerril-Villanueva E, Arreola R, Hernandez-Ferreira E, et al. IL-8 and IL-6 primarily mediate the inflammatory response in fibromyalgia patients. J Neuroimmunol 2016;290:22-5.

7. Correa-Rodriguez M, Casas-Barragan A, Gonzalez-Jimenez E, Schmidt-RioValle J, Molina F, Aguilar-Ferrandiz ME. Dietary inflammatory index scores are associated with pressure pain hypersensitivity in women with fibromyalgia. Pain Med 
2020;21:586-94.

8. Turkoglu G, Selvi Y. The relationship between chronotype, sleep disturbance, severity of fibromyalgia, and quality of life in patients with fibromyalgia. Chronobiol Int 2020;37:68-81.

9. Kocyigit BF, Okyay RA. The relationship between body mass index and pain, disease activity, depression and anxiety in women with fibromyalgia. PeerJ 2018;6:e4917.

10. Xiao Y, Haynes WL, Michalek JE, Russell IJ. Elevated serum high-sensitivity C-reactive protein levels in fibromyalgia syndrome patients correlate with body mass index, interleukin-6, interleukin-8, erythrocyte sedimentation rate. Rheumatol Int 2013;33:1259-64.

11. Backryd E, Tanum L, Lind AL, Larsson A, Gordh T. Evidence of both systemic inflammation and neuroinflammation in fibromyalgia patients, as assessed by a multiplex protein panel applied to the cerebrospinal fluid and to plasma. J Pain Res 2017;10:515-25.

12. Vincent A, Lahr BD, Wolfe F, Clauw DJ, Whipple MO, Oh TH, et al. Prevalence of fibromyalgia: a population-based study in Olmsted County, Minnesota, utilizing the Rochester Epidemiology Project. Arthritis Care Res (Hoboken) 2013;65:786-92. 13. Wolfe F, Ross K, Anderson J, Russell IJ, Hebert L. The prevalence and characteristics of fibromyalgia in the general population. Arthritis Rheum 1995;38:19-28.

14. Jones GT, Atzeni F, Beasley M, Fluss E, Sarzi-Puttini P, Macfarlane GJ. The prevalence of fibromyalgia in the general population: a comparison of the American College of Rheumatology 1990, 2010, and modified 2010 classification criteria. Arthritis Rheumatol 2015;67:568-75.

15. Walitt B, Nahin RL, Katz RS, Bergman MJ, Wolfe F. The prevalence and characteristics of fibromyalgia in the 2012 National Health Interview Survey. PLoS One 2015;10:e0138024. 16. Wolfe F, Hauser W, Hassett AL, Katz RS, Walitt BT. The development of fibromyalgia--I: examination of rates and predictors in patients with rheumatoid arthritis (RA). Pain 2011;152:291-9.

17. Lee YC, Lu B, Boire G, Haraoui BP, Hitchon CA, Pope JE, et al. Incidence and predictors of secondary fibromyalgia in an early arthritis cohort. Ann Rheum Dis 2013;72:949-54.

18. Karaaslan Y, Ozturk M, Haznedaroglu S. Secondary fibromyalgia in Turkish patients with rheumatologic disorders. Lupus 1999;8:486.

19. Jobanputra C, Richey RH, Nair J, Moots RJ, Goebel A. Fibromyalgia in Behcet's disease: a narrative review. Br J Pain 2017;11:97-101.

20. Lee SS, Yoon HJ, Chang HK, Park KS. Fibromyalgia in Behcet's disease is associated with anxiety and depression, and not with disease activity. Clin Exp Rheumatol 2005;23(4 Suppl 38):S15-9.

21. Toprak M, Erden M, Alpayci M, Ediz L, Yazmalar L, Hiz O, et al. The frequency and effect of fibromyalgia in patients with Behcet's disease. Turk J Phys Med Rehabil 2017;63:160-4.

22. Criteria for diagnosis of Behcet's disease. International Study Group for Behcet's Disease. Lancet 1990;335:1078-80.

23. Bennett RM, Friend R, Marcus D, Bernstein C, Han BK, Yachoui R, et al. Criteria for the diagnosis of fibromyalgia: val- idation of the modified 2010 preliminary American College of Rheumatology criteria and the development of alternative criteria. Arthritis Care Res (Hoboken) 2014;66:1364-73.

24. Burckhardt CS, Clark SR, Bennett RM. The fibromyalgia impact questionnaire: development and validation. J Rheumatol 1991;18:728-33.

25. Altan L, Celiker R, Ercan I, Birtane M, Akgun K, Zateri C, et al. The reliability and validity of the Turkish version of the Fibromyalgia Participation Questionnaire. Eur J Rheumatol 2018;5:40-4.

26. Wolfe F, Smythe HA, Yunus MB, Bennett RM, Bombardier C, Goldenberg DL, et al. The American College of Rheumatology 1990 Criteria for the Classification of Fibromyalgia. Report of the Multicenter Criteria Committee. Arthritis Rheum 1990;33:160-72.

27. de Araujo AL, Paliares IC, de Araujo MI, Novo NF, Cadaval RA, Martinez JE. [The association of fibromyalgia and systemic lupus erythematosus change the presentation and severity of both diseases?]. Rev Bras Reumatol 2015;55:37-42. [Article in Portuguese]

28. Jiao J, Davis III JM, Cha SS, Luedtke CA, Vincent A, Oh TH. Association of rheumatic diseases with symptom severity, quality of life, and treatment outcome in patients with fibromyalgia. Scand J Rheumatol 2016;45:49-56.

29. Gunturk EE, Ekiz T, Akkaya H. Evaluation of carotid-femoral pulse wave velocity, aortic stiffness index, and aortic distensibility in patients with fibromyalgia. Clin Rheumatol 2019;38:1731-5.

30. Ranzolin A, Duarte AL, Bredemeier M, da Costa Neto CA, Ascoli BM, Wollenhaupt-Aguiar B, et al. Evaluation of cytokines, oxidative stress markers and brain-derived neurotrophic factor in patients with fibromyalgia - A controlled cross-sectional study. Cytokine 2016;84:25-8.

31. Woolf CJ. Central sensitization: implications for the diagnosis and treatment of pain. Pain 2011;152(3 Suppl):S2-15.

32. Ostuni P, Botsios C, Sfriso P, Bertagnin A, Cozzi F, Doria A, et al. [Prevalence and clinical features of fibromyalgia in systemic lupus erythematosus, systemic sclerosis and Sjogren's syndrome]. Minerva Med 2002;93:203-9. [Article in Italian]

33. Kukidome D, Nishikawa T, Sato M, Igata M, Kawashima J, Shimoda S, et al. Measurement of small fibre pain threshold values for the early detection of diabetic polyneuropathy. Diabet Med 2016;33:62-9.

34. Massara A, Bonazza S, Castellino G, Caniatti L, Trotta F, Borrelli M, et al. Central nervous system involvement in Sjogren's syndrome: unusual, but not unremarkable--clinical, serological characteristics and outcomes in a large cohort of Italian patients. Rheumatology (Oxford) 2010;49:1540-9.

35. Akman-Demir G, Serdaroglu P, Tasci B. Clinical patterns of neurological involvement in Behcet's disease: evaluation of 200 patients. The Neuro-Behcet Study Group. Brain 1999;122(Pt 11):2171-82.

36. Jaskolska M, Chylinska M, Masiak A, Nowicka-Sauer K, Sieminski M, Zietkiewicz M, et al. Peripheral neuropathy and health-related quality of life in patients with primary Sjogren's syndrome: a preliminary report. Rheumatol Int 2020;40:1267-74. 
37. Melikoglu M, Melikoglu MA. The prevalence of fibromyal- activity. Rheumatol Int 2013;33:1219-22. gia in patients with Behcet's disease and its relation with disease 